\title{
Reduced Mechanism Approach of Modeling Premixed Propane-Air Mixture Using ANSYS Fluent
}

\author{
Lucky Anetor $^{1, *}$, Edward Osakue ${ }^{2}$, and Christopher Odetunde ${ }^{3}$ \\ 1 Department of Mechanical Engineering, Nigerian Defence Academy, Kaduna, Nigeria \\ 2 Department of Industrial Technology, Texas Southern University, Houston, Texas, USA \\ 3 Department of Aviation Science and Technology, Texas Southern University, Houston, Texas, \\ USA \\ E-mail: anetor55@yahoo.com*
}

Abstract. In combustion computational analysis, reduced mechanisms are often used in place of detailed kinetic chemistry. Since the computational costs of including all the species in the reactor model are always prohibitively high, several reduced mechanisms have been developed for propane and other hydrocarbon oxidation. In this study we employed ANSYS Fluent Computational Fluid Dynamics (CFD) package, (hereinafter referred to as Fluent) to analyze propane oxidation mechanism in a conical reactor. The $k-\varepsilon$ scheme was used to model the effects of turbulence. The reaction kinetics employed in this study is that based on the work of Westbrook and Dryer [14]. This simplified model consists of 5 chemical reactions and 12 species, namely, $\mathrm{C}_{3} \mathrm{H}_{8}, \mathrm{CO}_{2}$, $\mathrm{CO}, \mathrm{H}_{2} \mathrm{O}, \mathrm{NO}, \mathrm{O}_{2}, \mathrm{O}, \mathrm{H}, \mathrm{N}, \mathrm{OH}, \mathrm{N}_{2}$ and $\mathrm{H}_{2}$. The computed mass fractions of the species, $\mathrm{C}_{3} \mathrm{H}_{8}, \mathrm{CO}_{2}, \mathrm{CO}$ and $\mathrm{H}_{2} \mathrm{O}$ were found to be in agreement measured values presented in [20]. The results show that the bulk of the turbulent kinetic energy was produced in the inlet jet. The computed values of $y^{*}$ were found to confirm that the use of the law-of-the-wall functions was valid and also showed that the computational mesh for the present model was appropriate.

Keywords: Chemical kinetics, reaction mechanism, ignition delays, flame speed turbulent kinetic energy, energy and combustion devices.

ENGINEERING JOURNAL Volume 16 Issue 1

Received 20 September 2011

Accepted 25 October 2011

Published 1 January 2012

Online at http://www.engj.org

DOI:10.4186/ej.2012.16.1.67 


\section{Introduction}

The growing cost of experimental tests in industrial combustors and the efforts to reduce development time, has led power plant designers to develop computer programs which are capable of predicting the performance of the combustors. The contributions made by these computational methods have become all the more important because of the increase in severity in engine cycle variability and the requirements for reducing pollution in automobiles, aircrafts, furnaces, etc. These power plants require different types of combustor design and various computational methods have been used to carry out investigations on them.

In many of the combustion models used in computational methods, chemical kinetics is reduced to a single-step empirical expression or to global multi-step schemes. However, a better knowledge of the chemical kinetics of combustion is essential for the quality of the prediction of many of the important phenomena such as ignition delays, flame speed, blow-out, engine efficiency and pollutant emissions. In view of this, several studies in the field of chemical kinetics have been devoted to the conception of reaction mechanisms with growing complexity for the representation of hydrocarbon oxidation.

Because of the superiority of detailed chemical kinetic mechanisms to predict combustion chemistry features, many researchers have begun to develop detailed mechanisms for the oxidation of higher hydrocarbons in a wide range of temperatures, pressures and equivalence ratios. However, very large mechanisms cannot be introduced into complex multi-dimensional computational fluid dynamics (CFD) codes for the modeling of the combustion of practical fuels. For this reason, efforts are been made to employ reduction techniques for the simplification of large mechanisms in order to keep the essential features of the chemistry in realistic combustor configurations.

This study focuses on the combustion of premixed propane-air mixture for several reasons. First and foremost, the ignition properties of propane resemble those of other higher hydrocarbons but are different from those of the lower hydrocarbons (e.g. methane, ethylene and acetylene). Propane, therefore, may be the smallest in the homologous series of hydrocarbons that is representative of higher hydrocarbons in ignition and detonation characteristics. Since the overall activation energy and ignition times of propane are similar to those of other higher hydrocarbons, including liquid fuels that are suitable for many industrial applications, propane is always chosen as a model fuel for several numerical and experimental studies.

This study consists of seven sections. Section 1 briefly introduces the present study, section 2 presents a literature review of some reduced hydrocarbon mechanisms, the objective of the present study is stated in section 3 . In section 4, relevant theory of species transport and finite-rate chemistry are presented, while section 5 presents the definition of the problem to be addressed, boundary conditions and the details of the numerical solution. The results of this study are presented in section 6 and finally, section 7 presents a summary and conclusions of this study.

\section{Reduced Mechanism}

Extensive research efforts aimed at addressing the issues mentioned above are presently vigorously pursued. One of the most critical areas been looked at is the chemical kinetics or the mechanisms responsible for combustion processes. Chemical kinetics is predominantly used in the modeling of complex chemical processes [1].

The chemistry of several reacting systems is often described by detailed mechanisms that consist of a large number of species, $N_{s}$, and elementary reactions, $N_{r}$. Examples can be drawn from fields as diverse as combustion, polymerization, environmental science, materials technology, and microelectronics processing. To establish the material and energy balances for these systems in a reactor, the number of component mass balances required is $N_{s}$. Depending on the type of reactor, these balances could involve nonlinear algebraic equations, ordinary differential equations, partial differential equations, or some combination of these equations.

The computer processing resources required to solve these problems increase exponentially with increase in $N_{s}$. This difficulty and expense have motivated the development of reduced or simplified mechanisms that retain only a limited set of species and reactions necessary to capture the essential features of combustion chemistry as long as the simulated mechanism matches the predictions of laminar flame speed, adiabatic temperature rise, auto-ignition delay time, extinction time, and 
concentration profiles of key species within a pre-specified accuracy. Once obtained, the reduced mechanism can be directly incorporated into the chemical source terms of a reactor model having any level of complexity.

Through application of steady state and partial equilibrium assumptions, these reduced mechanisms describing fuel oxidation, and nitrogen chemistry typically consist of four to ten lumped steps. Among the lumped reactions, there are no more than four to six global reactions for hydrocarbon oxidation. Performance validations show that they are indeed descriptive of situations under which the relevant assumptions are valid though the performance can substantially deteriorate for other situations. There exists, therefore, the need to develop reduced mechanisms that are sufficiently comprehensive in capability to describe as wide a range of combustion phenomena and situations as possible. The penalty associated with robustness of reduced mechanisms is the increase in size.

The techniques for a priori mechanism reduction fall into three major categories which are: quasisteady-state partial-equilibrium hypothesis [2]; time-scale analysis [3] and [4] and mathematical programming methods, [5], [6], and [7]. These referenced studies employed a simplified reactor model such as batch, plug-flow, or perfectly stirred tank to study the relative influence of individual reactions on the features of the combustion process. Transport effects are neglected while performing the reduction, but their influence is, nonetheless, and often checked a posteriori by testing the full and reduced mechanisms on a simplified model that includes these effects. A comprehensive review of reduction techniques for kinetic models with an emphasis on combustion can be found in [8], [9], [10], [11] and [12].

Propane is a component of natural gas which can be liquefied at high pressures. Propane is also a clean and easily-transportable fuel. It is similarly used as fuel for jet engines and in the form of liquefied petroleum gas (LPG) for car engines. For the present study, the chemical kinetics of propane is investigated using the Fluent CFD code.

\section{Objective of the Present Study}

As stated above, the purpose of this study is to use the ANSYS Fluent CFD package [13] to model premix propane-air oxidation in a conical reactor using the model based on the work published by Westbrook and Dryer [14]. This simplified model consists of 5 chemical (forward and backward) reactions and 12 species. The forward and backward reactions were considered in the present study.

The five-step (forward and backward) scheme of Westbrook and Dryer [14] is given below:

$$
\begin{aligned}
\mathrm{C}_{3} \mathrm{H}_{8}+3.5 \mathrm{O}_{2} \rightarrow & 3 \mathrm{CO}+4 \mathrm{H}_{2} \mathrm{O} \\
k_{f} & =10^{17.25} \exp (-15106 / \mathrm{T})\left[\mathrm{C}_{3} \mathrm{H}_{8}\right]^{0.1}\left[\mathrm{O}_{2}\right]^{1.65} \\
k_{b}= & 0 \\
\mathrm{CO}+0.5 \mathrm{O}_{2} \rightarrow & C \mathrm{O}_{2} \\
k_{f}= & 10^{19.85} \exp (-20142 / \mathrm{T})[\mathrm{CO}]^{1}\left[\mathrm{H}_{2} O\right]^{0.5}\left[\mathrm{O}_{2}\right]^{0.25} \\
k_{b}= & 5 \times 10^{11} \exp (-20142 / \mathrm{T})[\mathrm{CO}]^{1.0} \\
O_{2}+2 N_{2} \rightarrow 2 N & +2 N O \\
k_{f}= & 1.5587 \times 10^{14} \exp (-67627 / R T)[C O]^{1}\left[H_{2} O\right]^{1.0}\left[O_{2}\right]^{2} \\
k_{b}= & 7.5 \times 10^{12} \exp (0)\left[O_{2}\right]^{1.0}[N]^{2}[N O]^{2} \\
2 O_{2}+N_{2} \rightarrow & 2 O+2 N O \\
k_{f}= & 2.6484 \times 10^{19 .} \exp (-59418 / T)\left[O_{2}\right]^{2.0}\left[N_{2}\right]^{1} \\
k_{b}= & 1.6 \times 10^{21} \exp (-19678 / T)[O]^{2}[N O]^{2}
\end{aligned}
$$




$$
\begin{aligned}
N_{2}+2 O H & \rightarrow 2 H+2 N O \\
k_{f} & =2.123 \times 10^{14} \exp (-57020 / T)[N]^{2}[O H]^{2} \\
k_{b} & =0
\end{aligned}
$$

where the forward and backward reaction rates, $k_{f}$ and $k_{b}$ respectively are expressed in $\mathrm{kmol} / \mathrm{m}^{3}-s$, the temperature, $T$ is in Kelvin $(\mathrm{K})$ and $\Phi=0.90$ is the equivalence ratio.

\section{Theory of Species Transport and Finite-Rate Chemistry}

Fluent can be used to model the mixing and transport of chemical species by solving the conservation equations describing convection, diffusion, and reaction sources for each component species. Multiple simultaneous chemical reactions can be modeled, with reactions occurring in the bulk phase (volumetric reactions) and/or on wall or particle surfaces, and in the porous region. Species transport modeling capabilities, both with and without reactions, are described in this section.

\subsection{Volumetric Reactions - Species Transport Equations}

Theoretical information about species transport and finite-rate chemistry as related to volumetric reactions is presented in this section.

In order to solve the conservation equations for chemical species, Fluent predicts the local mass fraction of each species, $Y_{i}$, through the solution of a convection-diffusion equation for the $i^{\text {th }}$ species. This conservation equation takes the following general form:

$$
\frac{\partial}{\partial t}\left(\rho Y_{i}\right)+\nabla \cdot\left(\rho \vec{v} Y_{i}\right)=-\nabla \cdot \vec{J}_{i}+R_{i}+S_{i}
$$

$R_{i}$ is the net rate of production of species $i$ by chemical reaction and $S_{i}$ is the rate of creation by addition from the dispersed phase plus any user-defined sources. An equation of this form will be solved for $N-1$ species where $N$ is the total number of fluid phase chemical species present in the system. Since the mass fraction of the species must sum to unity, the $N^{\text {th }}$ mass fraction is determined as one minus the sum of the $N-1$ solved mass fractions. To minimize numerical error, the $N^{\text {th }}$ species should be selected as that species with the overall largest mass fraction, such as $N_{2}$ when the oxidizer is air.

\subsection{Mass Diffusion in Laminar Flows}

In Eq. (6), $\vec{J}_{i}$ is the diffusion flux of species $i$, which arises due to gradients of concentration and temperature. By default, Fluent uses the dilute approximation (also called Ficks law of diffusion) to model mass diffusion due to concentration gradients, under which the diffusion flux can be written as:

$$
\vec{J}_{i}=-\rho D_{i, m} \nabla Y_{i}-D_{T, i} \frac{\nabla T}{T}
$$

$D_{i, m}$ is the mass diffusion coefficient for species $i$ in the mixture, and $D_{T, i}$ is the thermal (Soret) diffusion coefficient. For certain laminar flows, the dilute approximation may not be acceptable, and full multi-component diffusion is instead required and in such cases, the Maxwell-Stefan equations are solved.

\subsection{Mass Diffusion in Turbulent Flows}

In turbulent flows, Fluent computes the mass diffusion in the following form:

$$
\vec{J}_{i}=-\left(\rho D_{i, m}+\frac{\mu_{t}}{S c_{t}}\right) \nabla Y_{i}-D_{T, i} \frac{\nabla T}{T}
$$


$S c_{t}$ is the turbulent Schmidt number $\left(\mu_{t} / \rho D_{t}\right.$, where $\mu_{t}$ is the turbulent viscosity and $D_{t}$ is the turbulent diffusivity). The default $S c_{t}$ number is 0.7 . It is worth noting that turbulent diffusion generally overwhelms laminar diffusion and, for this reason, specification of detailed laminar diffusion properties in turbulent flows becomes superfluous.

\subsection{Treatment of Species Transport in the Energy Equation}

For many multi-component mixing flows, the transport of enthalpy due to species diffusion is:

$$
\nabla \cdot\left[\sum_{i=1}^{n} h_{i} \vec{J}_{i}\right]
$$

The transport of enthalpy can have a significant effect on the enthalpy field and should, therefore, not be neglected in the analysis whenever the Lewis number for any specie is far from unity. Neglecting this term can lead to significant errors. Fluent includes this term by default. In Eq. (9), $k$ is the thermal conductivity.

\subsection{Diffusion at Inlets}

$$
L_{e i}=\frac{k}{\rho c_{p} D_{i, m}}
$$

For the pressure-based solver in Fluent, the net transport of species at inlets consists of both convection and diffusion components. (For the density-based solvers, only the convection component is included.) The convection component is fixed by the inlet species mass fraction specified by the user. The diffusion component, however, depends on the gradient of the computed species field at the inlet. Thus the diffusion component (and therefore the net inlet transport) is not specified a priori.

\subsection{The Generalized Finite-Rate Formulation for Reaction Modeling}

The reaction rates that appear as source terms in Eq. (6) are computed in Fluent, for turbulent flows, by one of three models:

- Laminar finite-rate model: The effects of turbulent fluctuations are ignored, and reaction rates are determined by Arrhenius kinetic expressions.

- Eddy-dissipation model: Reaction rates are assumed to be controlled by the turbulence, so expensive Arrhenius chemical kinetic calculations can be avoided. The model is computationally cheap, but, for realistic results, only one or two step heat-release mechanisms should be used.

- Eddy-dissipation-concept (EDC) model: Detailed Arrhenius chemical kinetics can be incorporated in turbulent flames. It is worth noting that detailed chemical kinetic calculations are computationally expensive.

The generalized finite-rate formulation is suitable for a wide range of applications including laminar or turbulent reaction systems, and combustion systems with premixed, non-premixed, or partially premixed flames.

\subsection{The Laminar Finite-Rate Model}

The laminar finite-rate model computes the chemical source terms using Arrhenius expressions, and ignores the effects of turbulent fluctuations. The model is exact for laminar flames, but is generally inaccurate for turbulent flames due to highly non-linear Arrhenius chemical kinetics. The laminar model may, however, be acceptable for combustion with relatively slow chemistry and small turbulence-chemistry interaction, such as supersonic flames.

The net source of chemical species $i$ due to reaction is computed as the sum of the Arrhenius reaction sources over the $N_{R}$ reactions that the species participate in:

$$
R_{i}=M_{w, i} \sum_{r=1}^{N_{R}} \hat{R}_{i, r}
$$


where $M_{w, i}$ is the molecular weight of species $i$ and $\hat{R}_{i, r}$ is the Arrhenius molar rate of creation/destruction of species $i$ in reaction $r$. Reaction may occur in the continuous phase at wall surfaces.

Consider the $r$ th reaction written in general form as follows:

$$
\sum_{r=1}^{N} v_{i, r}^{\prime} M_{i} \underset{k_{f, b}}{\stackrel{k_{f, r}}{\Leftrightarrow}} \sum_{r=1}^{N} v_{i, r}^{\prime \prime} M_{i}
$$

where

$N=$ number of chemical species in the system;

$v_{i, r}^{\prime}=$ stoichiometric coefficient for reactant $i$ in reaction $r$;

$v^{\prime \prime}{ }_{i, r}=$ stoichiometric coefficient for product $i$ in reaction $r$

$M_{i}=$ symbol denoting species $i$

$k_{f, r}=$ forward rate constant for reaction $r$;

$k_{b, r}=$ backward rate constant for reaction $r$;

Equation (11) is valid for both reversible and non-reversible reactions. (Reactions in Fluent are non-reversible by default.) For non-reversible reactions, the backward rate constant, $k_{b, r}$, is simply omitted.

The summations in Eq. (11) are for all chemical species in the system, but only species that appear as reactants or products will have non-zero stoichiometric coefficients. Hence, species that are not involved will drop out of the equation.

For a non-reversible reaction for which the Backward Reaction button is disabled, the molar rate of creation/destruction, $\left(\widehat{R}_{i, r}\right)$ in Eq. (10) of species $i$ in reaction $r$ is given by

$$
\widehat{R}_{i, r}=\Gamma\left(v_{i, r}^{\prime \prime}-v_{i, r}^{\prime}\right)\left(k_{f, r} \prod_{j=1}^{N}\left[C_{j, r}\right]^{\left(\eta_{j, r}^{\prime}+\eta_{j, r}^{\prime \prime}\right)}\right)
$$

where

$C_{j, r}=$ molar concentration of species $j$ in reaction $r\left(\mathrm{kgmol}^{3} \mathrm{~m}^{3}\right)$

$\eta_{j, r}^{\prime}=$ rate exponent for reactant species $j$ in reaction $r$

$\eta^{\prime \prime}{ }_{j, r}=$ rate exponent for product species $j$ in reaction $r$.

For a reversible reaction, the molar rate of creation/destruction, $\left(\widehat{R}_{i, r}\right)$ of species $i$ in reaction $r$ is given by

$$
\widehat{R}_{i, r}=\Gamma\left(v_{i, r}^{\prime \prime}-v_{i, r}^{\prime}\right)\left(k_{f, r} \prod_{j=1}^{N}\left[C_{j, r}\right]^{\eta_{j, r}^{\prime}}-k_{b, r} \prod_{j=1}^{N}\left[C_{j, r}\right]^{\eta_{j, r}^{\prime \prime}}\right)
$$

Note that the rate exponent for the reverse reaction part in Eq. (13) is always the product species stoichiometric coefficient $\left(v_{j, r}{ }_{j, r}\right)$.

$\Gamma$ represents the net effect of third bodies on the reaction rate. $\Gamma$ is given by

$$
\Gamma=\sum_{j}^{N} \gamma_{j, r} C_{j}
$$

where $\gamma_{j, r}$ is the third-body efficiency of the $j$ th species in the $r$ th reaction. By default, Fluent does not include third-body effects in the reaction rate calculation. The effect of third-body efficiencies can be included if the data for them are available.

The forward rate constant for reaction $r, k_{f, r}$, is computed using the Arrhenius expression

$$
k_{f, r}=A_{r} T^{\beta_{r}} e^{-E_{r} / R T}
$$

where

$A_{r}=$ pre-exponential factor (consistent units)

$\beta_{r}=$ temperature exponent (dimensionless)

$E_{r}=$ activation energy for the reaction $(\mathrm{J} / \mathrm{kmol})$

$R=$ universal gas constant $(\mathrm{J} / \mathrm{kmol}-K)$.

The values of $v_{i, r}^{\prime} v_{i, r}^{\prime \prime}, \eta_{j, r}^{\prime}, \eta_{j, r}^{\prime \prime}, \beta_{r}, A_{r}$, and $E_{r}$ is usually specified during problem definition. 
If the reaction is reversible, the backward rate constant for reaction $r, k_{b, r}$, is computed from the forward rate constant using the following relation:

$$
k_{b, r}=\frac{k_{f, r}}{k_{r}}
$$

where $K_{r}$ is the equilibrium constant for the $r$ th reaction, computed from

$$
k_{r}=\exp \left(\frac{\Delta S_{r}^{0}}{R}-\frac{\Delta H_{r}^{0}}{R T}\right)\left(\frac{p_{a t m}}{R T}\right)^{\sum_{i=1}^{N}\left(v_{i, r}^{\prime \prime}-v_{i, r}^{\prime}\right)}
$$

$p_{a t m}$ denotes atmospheric pressure $(101325 \mathrm{~Pa})$. The term within the exponential function represents the change in Gibbs free energy, and its components are computed as follows:

$$
\begin{gathered}
\frac{\Delta S_{r}^{0}}{R}=\sum\left(v_{i, r}^{\prime \prime}-v_{i, j}^{\prime}\right) \frac{S_{i}^{0}}{R} \\
\frac{\Delta H_{r}^{0}}{R T}=\sum\left(v_{i, r}^{\prime \prime}-v_{i, j}^{\prime}\right) \frac{h_{i}^{0}}{R T}
\end{gathered}
$$

where $S_{i}^{0}$ and $h_{i}^{0}$ are the standard-state entropy and standard-state enthalpy (heat of formation). These values are specified in Fluent as properties of the mixture material.

\subsection{The Eddy-Dissipation Model}

Most fuels are fast burning, and the overall rate of reaction is controlled by turbulent mixing. In nonpremixed flames, turbulence slowly convects/mixes fuel and oxidizer into the reaction zones where they burn quickly. In premixed flames, the turbulence slowly convects/mixes cold reactants and hot products into the reaction zones, where reaction occurs rapidly. In such cases, the combustion is said to be mixing-limited, and the complex and often unknown, chemical kinetic rates can be safely neglected.

Fluent provides a turbulence-chemistry interaction model, based on the work of Magnussen and Hjertager [15], called the eddy-dissipation model. The net rate of production of species $i$ due to reaction $r, R_{i, r}$, is given by the smaller (i.e., limiting value) of the two equations below:

$$
\begin{gathered}
R_{i, r}=v_{i, j}^{\prime} M_{w, i} A \rho \frac{\varepsilon}{k} \min _{R}\left(\frac{Y_{R}}{v_{i, r}^{\prime} M_{w, R}}\right) \\
R_{i, r}=v_{i, j}^{\prime} M_{w, i} A B \rho \frac{\varepsilon}{k}\left(\frac{\sum_{P} Y_{P}}{\sum_{j}^{N} v_{j, r}^{\prime \prime} M_{w, j}}\right)
\end{gathered}
$$

where

$Y_{P}$ is the mass fraction of any product species, $P$;

$Y_{R}$ is the mass fraction of a particular reactant, $R$;

$A$ is an empirical constant equal to 4.0 ;

$B$ is an empirical constant equal to 0.5 ;

$\varepsilon$ is the turbulent dissipation rate $\left(\mathrm{m}^{2} \mathrm{~s}^{-3}\right)$;

$k$ is the turbulent kinetic energy per unit mass $(\mathrm{J} / \mathrm{kg})$.

In Eq. (20) and (21), the chemical reaction rate is governed by the large-eddy mixing time scale, $k / \varepsilon$, as in the eddy-breakup model of Spalding [16]. Combustion proceeds whenever turbulence is present $(k / \varepsilon>0)$, and an ignition source is not required to initiate combustion. This is usually acceptable for non-premixed flames, but in premixed flames, the reactants will burn as soon as they enter the computational domain, upstream of the flame stabilizer. To remedy this, Fluent provides the finite-rate/eddy-dissipation model, where both the Arrhenius, Eq. (13), and eddy dissipation, Equations (20) and (21) reaction rates are calculated. The net reaction rate is taken as the minimum of these two rates. In practice, the Arrhenius rate acts as a kinetic switch, preventing reaction before the flame holder. Once the flame is ignited, the eddy-dissipation rate is generally smaller than the Arrhenius rate, and reactions are mixing-limited. 
Although Fluent allows multi-step reaction mechanisms (number of reactions > 2) with the eddy dissipation and finite-rate/eddy-dissipation models, such approach will likely produce incorrect solutions because multi-step chemical mechanisms are based on Arrhenius rates, which differ for each reaction. In the eddy-dissipation model, every reaction has the same, turbulent rate, and therefore the model should be used only for one-step (reactant $\rightarrow$ product), or two-step (reactant $\rightarrow$ intermediate, intermediate $\rightarrow$ product) global reactions. The model cannot predict kinetically controlled species such as radicals. To incorporate multi-step chemical kinetic mechanisms in turbulent flows, use the EddyDissipation Concept (EDC) model.

The eddy-dissipation model requires products to initiate reaction, see Eq. (11). When the solution is initialized for steady flows, Fluent sets all species mass fractions to a maximum of the user specified initial value and 0.01 . This is usually sufficient to start the reaction. However, if a mixing solution converges first, where all product mass fractions are zero, there may be a need to patch products into the reaction zone to ignite the flame.

\section{Problem Definition}

The schematic diagram of the conical combustor and the computational mesh used for this study are shown in Figs. 1 and 2. A small nozzle at the center of the combustor introduces the lean premixed propane-air mixture (equivalence ratio, $\Phi=0.90$ ) at a speed of $75 \mathrm{~m} / \mathrm{s}$ and at inlet temperature of $750 \mathrm{~K}$. The ensuing combustion involves several complex reactions between $\mathrm{C}_{3} \mathrm{H}_{8}, \mathrm{CO}_{2}, \mathrm{CO}, \mathrm{H}_{2} \mathrm{O}, \mathrm{NO}, \mathrm{O}_{2}, \mathrm{O}$, $\mathrm{H}, \mathrm{N}, \mathrm{OH}, \mathrm{N}_{2}$, and $\mathrm{H}_{2}$. The high-speed flow reverses direction in the combustor and exits through the co-axial outlet. The steps used to model this problem are described below. The results are also discussed below.

\subsection{Computational Domain and Mesh}

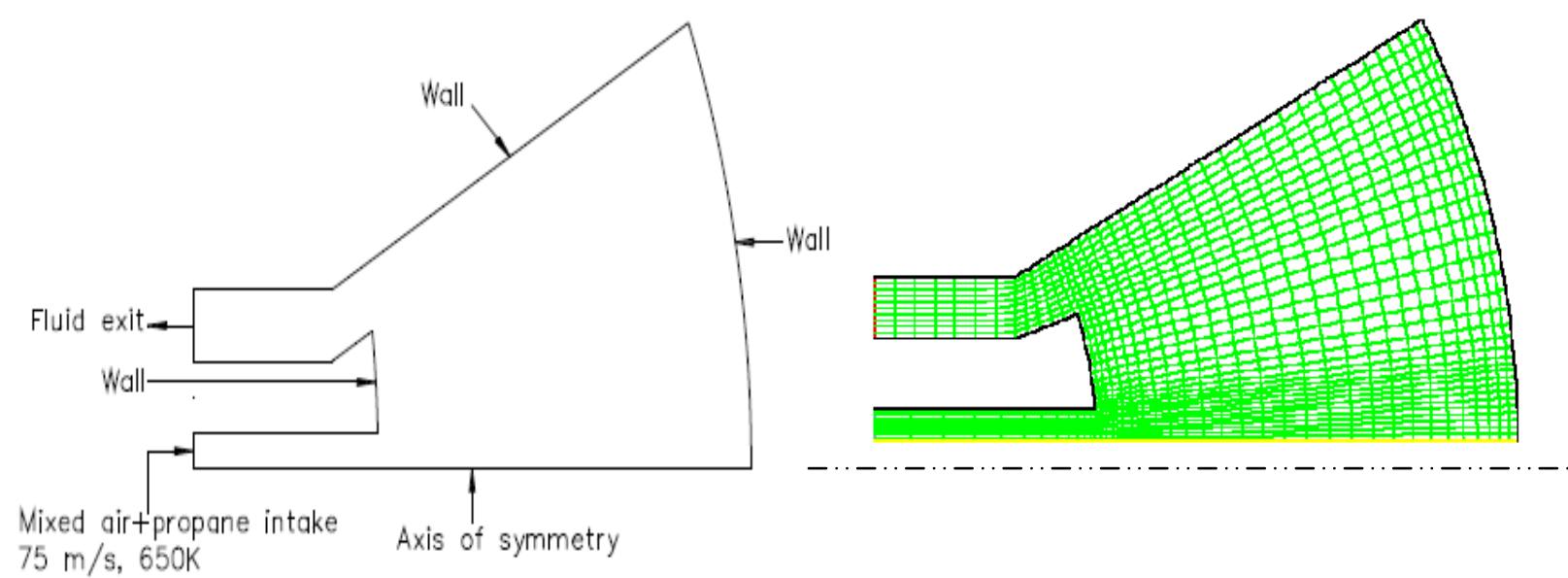

Fig. 1. Combustor geometry.

Fig. 2. Computational mesh.

In internal flows such as that inside a combustion chamber, the computational domain is the interior of the chamber. Because of the geometry of the combustion chamber, it was deemed appropriate to use a $2 D$ axisymmetric CFD model since the geometry of the actual $3 D$ combustion chamber is invariant in the third dimension below the plane of the inlet jet. We can see from the computational mesh that the mesh inside the inlet duct are more densely packed than elsewhere within the computational domain. This is to enable the proper resolution of the fast changing dynamics of the relevant quantities resulting from the high shear that is prevalent in this region.

The domain was divided into quadrilateral cells with a maximum cell squish of $9.96893 \times 10^{-2}$ and a maximum aspect ratio of 12.7911. A mesh size containing 1192 cells was found adequate for this problem. The computational mesh had a total of 2470 faces and 1279 nodes. The volume of the each control volume range from $1.288176 \times 10^{-10} \mathrm{~m}^{3}$ to $5.154941 \times 10^{-7} \mathrm{~m}^{3}$ for a total volume of $1.374440 \times$ $10^{-4} \mathrm{~m}^{3}$, while the face areas vary from $2.408730 \times 10^{-4} \mathrm{~m}^{2}$ to $3.825587 \times 10^{-3} \mathrm{~m}^{2}$. 
In order to reduce the bandwidth of the grid neighbor number and to speed up the computations, the "Reorder" feature of Fluent was used. This is very important for large cases involving 1 million or more nodes. The method used to reorder the computational domain is the Reverse Cuthill-McKee and it resulted in bandwidth reduction of $40 / 38=1.05$.

\subsection{Mathematical Modeling}

The sub-models used in this study are briefly described in this section.

\section{Turbulence Model}

The standard two-equation, $k-\varepsilon$ turbulence model with standard values was used for this study. At high Reynolds numbers the rate of dissipation of turbulent kinetic energy $\varepsilon$ is equal to the viscosity multiplied by the fluctuating vorticity. An exact transport equation for the fluctuating vorticity, and thus the dissipation rate, can be derived from the Navier-Stokes equation. The $k-\varepsilon$ turbulence model consists of the turbulent kinetic energy and dissipation equations given below:

$$
\begin{gathered}
\frac{\partial k}{\partial t}+\nabla \cdot(\rho \vec{u} k)=\nabla \cdot\left(\left[\mu_{\text {lam }}+\frac{\rho v_{t}}{\sigma_{k}}\right] \nabla k\right)+\rho v_{t} G-\rho \varepsilon \\
\frac{\partial \varepsilon}{\partial t}+\nabla \cdot(\rho \vec{u} \varepsilon)=\nabla \cdot\left(\left[\mu_{\text {lam }}+\frac{\rho v_{t}}{\sigma_{\varepsilon}}\right] \nabla \varepsilon\right)+C_{1 \varepsilon} \rho v_{t} G \frac{\varepsilon}{k}-C_{2 \varepsilon} \rho \frac{\varepsilon^{2}}{k}
\end{gathered}
$$

where $G$ represents the turbulent generation rate which is equal to

$$
G=2\left(\left[\frac{\partial u}{\partial x}\right]^{2}+\left[\frac{\partial v}{\partial y}\right]^{2}+\left[\frac{\partial w}{\partial z}\right]^{2}\right)+\left(\frac{\partial u}{\partial y}+\frac{\partial v}{\partial x}\right)^{2}+\left(\frac{\partial u}{\partial z}+\frac{\partial w}{\partial x}\right)^{2}+\left(\frac{\partial w}{\partial y}+\frac{\partial v}{\partial z}\right)^{2}
$$

In the implementation of this model the Kolmogorov-Prandtl expression for the turbulent viscosity, $v_{t}$ is used and it is given by:

$$
v_{t}=C_{\mu} \frac{\varepsilon^{2}}{k}
$$

In the equations above $\mathrm{C}_{\mu}, \sigma_{\mathrm{k}}, \sigma_{\epsilon}, \mathrm{C}_{1 \mu}$, and $\mathrm{C}_{2 \mu}$ are all taken to be constants and are given their usual standard values of: $0.09,1.0,1.3,1.44$ and 1.92 respectively.

\section{The Species Model}

The species were modeled using the model based on the work published by Westbrook and Dryer [14]. This simplified model consists of 5 chemical reactions and 12 species. The five reactions (forward and backward) together with their respective reaction constants are enumerated in section 3 above.

\section{Turbulent Gaseous Combustion Model}

The turbulence-chemistry interaction (gaseous combustion) was modeled using the finite-rate/eddy dissipation model. The model has been extensively described in section 4.8 above. Essentially, the net rate of reaction is the minimum of:

- The chemical production or depletion term (kinetic rate)

- The rate of dissipation of reactant eddies

- The rate of dissipation of product eddies 


\subsection{Boundary Conditions}

\section{Boundary Conditions at the Inlet Plane}

The boundary conditions at the inlet plane were specified as follows: Inlet velocity $=75 \mathrm{~m} / \mathrm{s}$, Turbulent intensity $=3.6 \%$, Turbulent length scale, $l=0.003 \mathrm{~m}$, Temperature $=750^{\circ} \mathrm{K}$; and species mass fractions, $C_{3} H_{8}=0.034, O 2=0.225$ and $N_{2}=0.741$.

\section{Outlet Boundary Conditions}

The boundary conditions at the outlet plane were specified as follows: Pressure, $P a=101325 \mathrm{~N} / \mathrm{m}^{2}$, Turbulent intensity $=3.6 \%$, Turbulent length scale, $l=0.003 \mathrm{~m}$, Back flow total temperature $=2500^{\circ} \mathrm{K}$; and species mass fractions $\mathrm{CO}_{2}=0.1, \mathrm{CO}=0.1, \mathrm{H}_{2} \mathrm{O}=0.1, \mathrm{NO}=0.1, \mathrm{O}_{2}=0.1$ and $\mathrm{N}_{2}=0.5$. For velocity coupling, coupled scheme was employed.

\section{Wall Boundary Conditions}

The thermal boundary condition was set at a fixed heat flux of zero (adiabatic condition), while the walls were set as not moving. The no slip conditions were prescribed for the wall shear stress.

The treatment of the boundary conditions concerned with turbulent flows was the same as for laminar flows except in the case of walls. The $k-\varepsilon$ turbulence model provides accurate solutions only for fully turbulent flows.

\section{Standard Wall Functions - Law-of-the-Wall}

In the part of the flow near to walls there exist regions in which the local Reynolds number of turbulence, $R e_{t}=k^{2} /(v \varepsilon)$, is so small that the viscous effects become more significant than the turbulent ones. In this viscous sublayer very steep gradients occur; so for accurate modeling many grid points would be required in this region. However, it is not necessary to discretize the $k$ and $\varepsilon$ conservation equations over this region as there exist suitable laws, called law-of-the-wall which relates the wall conditions to values of the dependent variables just outside the viscous sublayer.

In this study, the standard wall functions were used to model the near wall conditions. In Fluent the standard wall functions are based on the proposal of Launder and Spalding [17], and have been most widely used for industrial flows.

\section{Momentum}

The law of the wall for mean velocity gives:

$$
U^{*}=\frac{1}{\kappa} \ln \left(E y^{*}\right)
$$

where:

$$
\begin{aligned}
& U^{*} \equiv \frac{U_{P} C_{\mu}^{1 / 4} k_{P}^{1 / 2}}{\tau_{w} / \rho} \\
& y^{*}=\frac{\rho C_{\mu}^{1 / 4} k_{P}^{1 / 2} y_{P}}{\mu}
\end{aligned}
$$

and

$\kappa=$ Von Karman constant $(=0.4187)$

$E=$ Empurical constant $(=9.793)$

$U_{P}=$ Mean velocity of the fluid at point $\mathrm{P}$ 
$k_{P}=$ Turbulence kinetic energy at point $\mathrm{P}$

$y_{P}=$ Distance from point $\mathrm{P}$ to the wall

$\mu=$ Dynamic viscosity of the fluid.

The logarithmic law for mean velocity is known to be valid in the region just outside the sublayer. In this layer, we have: $30<y^{*}<300$. In Fluent, the log-law is employed when $y^{*}>11.225$.

When the mesh is such that $y^{*}<11.225$ at the wall-adjacent cells, Fluent applies the laminar stress-strain relationship:

$$
U^{*}=y^{*}
$$

It should be noted that, in Fluent, the laws-of-the-wall for mean velocity and temperature are based on the wall unit, $y^{*}$ rather than $y^{+} \equiv \rho u_{\tau} y / \mu$. These quantities are approximately equal in equilibrium turbulent boundary layers.

\section{Energy}

Reynolds' analogy between momentum and energy transport gives a similar logarithmic law for mean temperature. As in the law-of-the-wall for mean velocity, the law-of-the-wall for temperature employed in Fluent comprises of the following two different laws:

- linear law for the thermal conduction sublayer where conduction is important;

- logarithmic law for the turbulent region where effects of turbulence dominate conduction.

The thickness of the thermal conduction layer is, in general, different from the thickness of the (momentum) viscous sublayer, and changes from fluid to fluid. For example, the thickness of the thermal sublayer for a high-Prandtl-number fluid (e.g., oil) is much less than its momentum sublayer thickness. For fluids of low Prandtl numbers (e.g., liquid metal), on the contrary, it is much larger than the momentum sublayer thickness.

In highly compressible flows, the temperature distribution in the near-wall region can be significantly different from that of low subsonic flows, due to the heating by viscous dissipation. In Fluent, the temperature wall functions include the contribution from the viscous heating.

\section{Species}

When using wall functions for species transport, Fluent assumes that species transport behaves analogously to heat transfer.

\section{Turbulence}

In computational fluid dynamics (CFD), it is impossible to numerically simulate turbulence without discretizing the flow-field as far as the Kolmogorov micro-scales methodology referred to as direct numerical simulation (DNS). Because DNS simulations are exorbitantly expensive as a result of the memory required, computational and storage overheads, turbulence models are used to simulate the effects of turbulence. A variety of models are used, but generally turbulent kinetic energy is a fundamental flow property which must be calculated in order for fluid turbulence to be modeled.

\section{Reynolds-averaged Navier-Stokes equations}

Reynolds-averaged Navier-Stokes (RANS) simulations use the Boussinesq eddy viscosity hypothesis [18] to calculate the Reynolds stresses that result from the averaging procedure:

$$
u_{i}^{\prime} u_{j}^{\prime}=\frac{2}{3} k \delta_{i, j}-v_{t}\left(\frac{\partial \bar{u}_{i}}{\partial x_{j}}+\frac{\partial \bar{u}_{j}}{\partial x_{j}}\right)
$$

where, $v_{t}=c \cdot k^{\frac{1}{2}} l_{m}$ and $\delta_{i, j}$ is the Kronecker delta. 
The exact method of resolving turbulent kinetic energy depends upon the turbulence model used. The $k-\varepsilon$ models assume isotropy of turbulence whereby the normal stresses are equal, that is:

$$
u^{\prime 2}=v^{\prime 2}=w^{\prime 2}
$$

This assumption makes modeling of turbulence quantities; $k$ and $\varepsilon$, simpler but will not be accurate in scenarios where anisotropic behavior of turbulence stresses dominates. The implications of this in the production of turbulence leads to over-prediction as production depends on the mean rate of strain, and not on the difference between the normal stresses (as they are, in the assumption of isotropic turbulence) [17].

The Reynolds-stress models (RSM) use a different method to put a closure to the Reynolds stresses. With this approach the normal stresses are not assumed isotropic, so the issue with turbulent kinetic energy over-prediction is avoided.

\section{Boundary conditions - Inlet and Wall}

Boundary conditions are as important in CFD as the method of computing turbulent kinetic energy in order to correctly predict the flows, especially in high Reynolds number simulations. The methods employed in this study are given below:

For the inlet conditions, turbulent kinetic energy, $k$ is calculated as shown below:

$$
k=\frac{2}{3}(U I)^{2}
$$

where $\mathrm{I}$ is the initial turbulence intensity $(\%)$ and it is defined below. $\mathrm{U}=75 \mathrm{~m} / \mathrm{s}$, the initial inlet velocity magnitude and the inlet turbulent kinetic energy dissipation rate, $\varepsilon$, to be calculated from Eq. (33) below:

$$
\varepsilon=\frac{C_{\mu}^{3 / 4} k^{3 / 2}}{l}
$$

where $l(l=0.003 \mathrm{~m})$ is the turbulence or eddy length scale, which is defined below, and $C_{\mu}$ is a $(k-\varepsilon)$ model parameter whose value is typically given as 0.09 and $I=0.16 R e^{-1 / 8}$. Furthermore, $R e$ is the Reynolds number and $l=0.07 L$, with $L$ been a characteristic length scale. For internal flows this may take the value of the inlet duct (or pipe) width (or diameter) or the hydraulic diameter. In this study $L$ $(L=0.043 \mathrm{~m})$ was taken as the combustion chamber inlet diameter.

In the $k-\varepsilon$ turbulence model, the equation is solved in the whole domain including the walladjacent cells. The boundary condition for $k$ imposed at the wall is:

$$
\frac{\partial k}{\partial \vec{n}}=0
$$

where $\vec{n}$ is the local coordinate normal to the wall.

The production of kinetic energy, $G_{k}$, and its dissipation rate, $\varepsilon$, at the wall-adjacent cells are the source terms in the $k$ equation. These are computed on the basis of the local equilibrium hypothesis. Under this assumption, the production of $k$ and its dissipation rate are assumed to be equal in the walladjacent control volume.

Thus, the production of $k$ is computed from:

and $\varepsilon$ is computed from:

$$
G_{k} \approx \tau_{w} \frac{\partial U}{\partial y}=\frac{\tau_{w}}{\kappa \rho C_{\mu}^{1 / 4} k_{P}^{1 / 2} y_{P}}
$$

$$
\varepsilon_{P}=\frac{C_{\mu}^{3 / 4} k_{P}^{3 / 2}}{y_{P}}
$$

The $\varepsilon$ - equation is not solved at the wall-adjacent cells, instead, it is computed using Eq. (36).

Note that, the wall boundary conditions for the solution variables, including mean velocity, temperature, species concentration, $k$ and $\varepsilon$, are all taken care of by the wall functions. Therefore, we do not need to be concerned with boundary conditions at the walls. The standard wall functions described above were used in the present work. The standard wall functions work reasonably well for a broad range of wall-bounded flows; however, they tend to become less reliable when the flow situations 
depart away from the ideal conditions that are assumed in their derivation. Among others, the constantshear and local equilibrium hypotheses are the ones that most restrict the universality of the standard wall functions. Accordingly, when the near-wall flows are subjected to severe pressure gradients as well as when the flows are in strong non-equilibrium, the quality of the predictions is likely to be compromised. The non-equilibrium wall functions offered as an additional option can improve the results in such situations.

\subsection{Solution Procedures for Chemical Mixing and Finite-Rate Chemistry}

Although many simulations involving chemical species may require no special treatment during the solution process, however, situations do arise that one or more of the solution techniques noted in this section help(s) to accelerate the convergence or improve the stability of more complex simulations. It was observed that the techniques outlined in this section were highly relevant to the present study.

\section{a. Stability and Convergence in Reacting Flows}

Obtaining a converged solution in a reacting flow can be difficult for a number of reasons. First, the impact of the chemical reaction on the basic flow pattern may be strong, leading to a model in which there is strong coupling between the mass/momentum balances and the species transport equations. This is especially true in combustion, where the reactions lead to a large heat release and subsequent density changes and large accelerations in the flow. All reacting systems have some degree of coupling, especially when the flow properties depend on the species concentrations. These coupling issues are best addressed by the use of a two-step solution process and also by the use of under-relaxation.

A second convergence issue in reacting flows involves the magnitude of the reaction source term. The model presented in this work involves very rapid reaction rates (that is, much more rapid than the rates of convection and diffusion) for which the solution of the species transport equations becomes numerically difficult. Such systems are termed stiff systems and are created whenever models involve very rapid kinetic rates, especially when these rates describe reversible or competing reactions. In the eddy-dissipation model, the fast reaction rates are removed by using the slower turbulent rates. For non-premixed systems, reaction rates are eliminated from the model. For stiff systems with laminar chemistry, the coupled solver is recommended instead of the segregated solver. For a turbulent finiterate mechanism which can be stiff, the Eddy Dissipation Concept for which a stiff Ordinary Differential Equation integrator for the chemistry is used and always recommended.

\section{b. Two-Step Solution Procedure (Cold Flow Simulation)}

Solving a reacting flow as a two-step process could be a realistic method for reaching a stable converged solution to a simulation problem. In this process, we began by solving the flow, energy, and species equations with reactions disabled (the cold-flow, or an un-reacting flow). Once the basic flow field has been established, the reactions are turn-on and continue the calculation. The cold-flow solution provides a good starting solution for the calculation of the combusting system. This two-step approach to combustion modeling was employed in the present study and was accomplished as follows:

1. The problem was setup including all species and reactions of interest.

2. The reaction calculations were temporarily disabled by turning off Volumetric Reactions.

3. The calculation of the product species were turned off.

4. An initial (cold-flow) solution was calculated. It is pertinent to mention that, it is generally not a worthwhile venture to obtain a fully converged cold-flow solution unless the non-reacting solution is also of interest.

5. The reaction calculations were then enabled by turning on Volumetric Reactions.

6. All other equations were turned on at this point as well. Since we used the finite-rate/eddy dissipation model for turbulence-chemistry interaction, we had to patch an ignition source in order to initiate combustion - the way we achieved this is described below. 


\section{c. Ignition in Combustion Simulations}

It is a well known fact that if fuel is introduced to an oxidant, spontaneous ignition does not occur unless the temperature of the mixture exceeds the activation energy threshold required to maintain combustion. This physical issue manifested itself in the present simulation as well. As mentioned above, we used the finite-rate/eddy-dissipation model for turbulence-chemistry interaction; hence, it was necessary to supply an ignition source to initiate combustion. In the present study the initial spark was supplied by patching a high temperature field $\left(1500^{\circ} \mathrm{C}\right)$ into a region of the model that contained a sufficient premixed fuel/air/product mixture for ignition to occur. It is worth stating that the initial patch has no impact on the final steady-state solution - no more than the location of a match determines the final flow pattern of the fire/inferno it initiates.

\subsection{Solver}

The Pressure-Based coupled algorithm which is a good alternative to density-based solvers of ANSYS Fluent when dealing with applications involving high shear flow was employed.

The following spatial discretization schemes were used:

- For gradient - the least square cell based method was employed; and

- The second - order upwind scheme was employed for density, momentum, modified turbulent viscosity and the energy equations.

Under relaxation factor of 0.8 was used for the species, energy and density while a value of 0.6 was applied for the momentum, turbulent kinetic energy, turbulent kinetic dissipation rate and turbulent viscosity.

In order to monitor convergence as the calculations (iterations) progressed, the following residual quantities: continuity, $x$-velocity, $y$-velocity, energy, turbulent kinetic energy, $k$, turbulent dissipation rate, $\varepsilon$, and all the species were monitored. The absolute convergence criteria which were set for these quantities are: continuity $10^{-3}, x$-velocity $10^{-3}, y$-velocity $10^{-3}$ and energy $10^{-4}$ for cold flow calculations. For the reacting flow computations, the initial temperature field was patched to initiate the combustion process. The absolute convergence criteria for the species were then lowered to $10^{-6}$.

The initial calculations were performed assuming that all properties except density were constant. Using constant transport properties (viscosity, thermal conductivity, and mass diffusion coefficients) is acceptable here because the flow is fully turbulent. The molecular transport properties will play a minor role compared to turbulent transport. The assumption of constant specific heat, in contrast, has a strong effect on the combustion solution, and this property was represented with a polynomial function during the reacting flow computation.

The solution was then initialized. Thereafter, the Full Multigrid (FMG) feature was run. Full Multigrid initialization often facilitates an easier start-up, thereby, obviating the need for CourantFriedrichs-Lewy (CFL) condition ramping, consequently reducing the number of iterations required for convergence.

\section{Discussion of Results}

Figures 1 and 2 above show the schematic diagrams of the conical combustion chamber and the computational mesh respectively.

The simulation code was first run for 200 iterations, and subsequently for 500 and 1100 iterations and the scaled residuals are shown in Figs. 3 and 4 respectively. Since it is generally not a worthwhile venture to obtain a fully converged cold-flow solution, it was decided to run the cold-flow calculations for only 200 iterations in the first instance. As can be seen in Fig. 3, the solution converged rapidly because there were no large temperature fluctuations that would cause sharp gradient of quantities, especially those susceptible to large temperature fluctuation like density. 


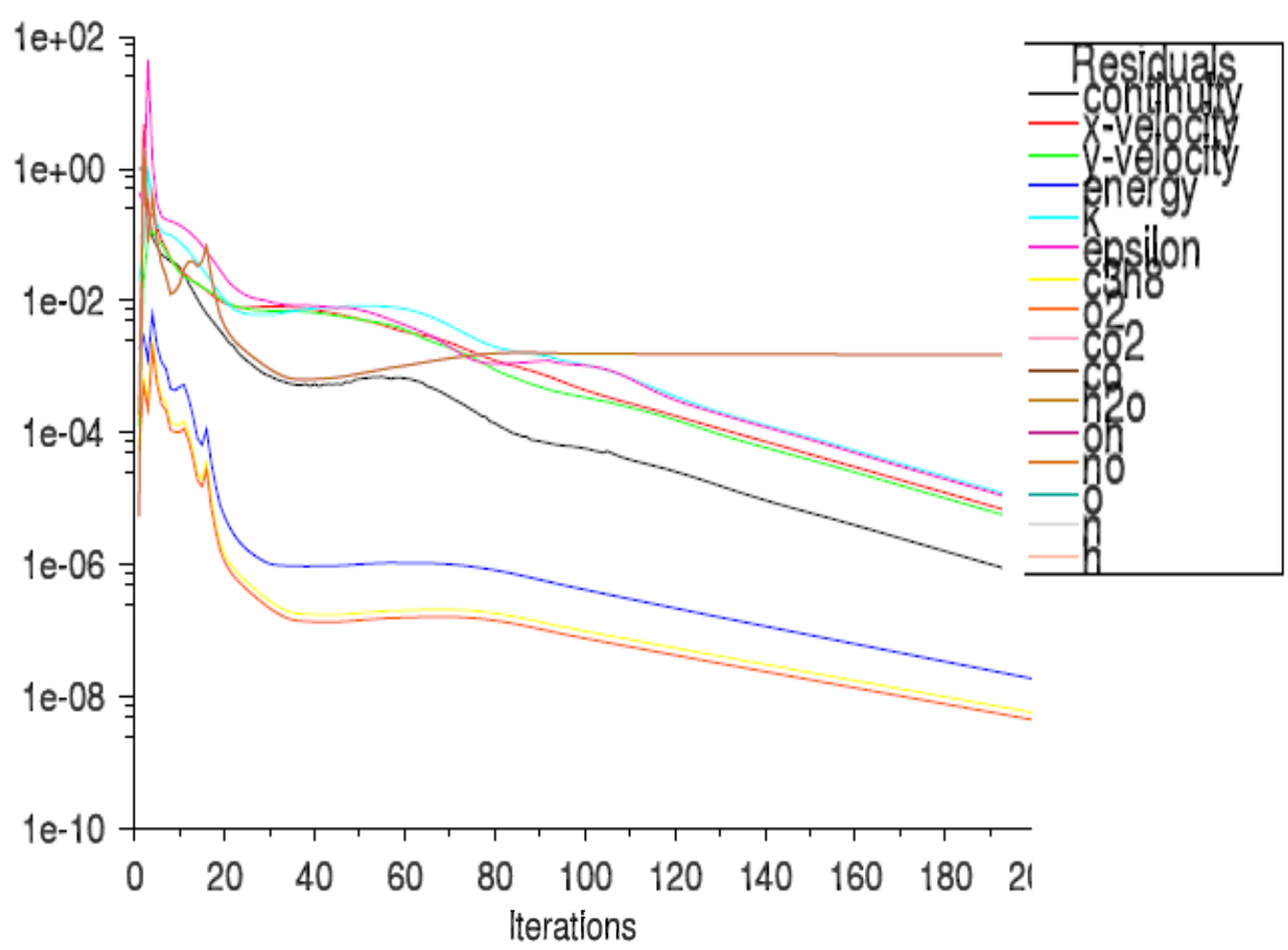

Fig. 3. Residual after 200 iterations.

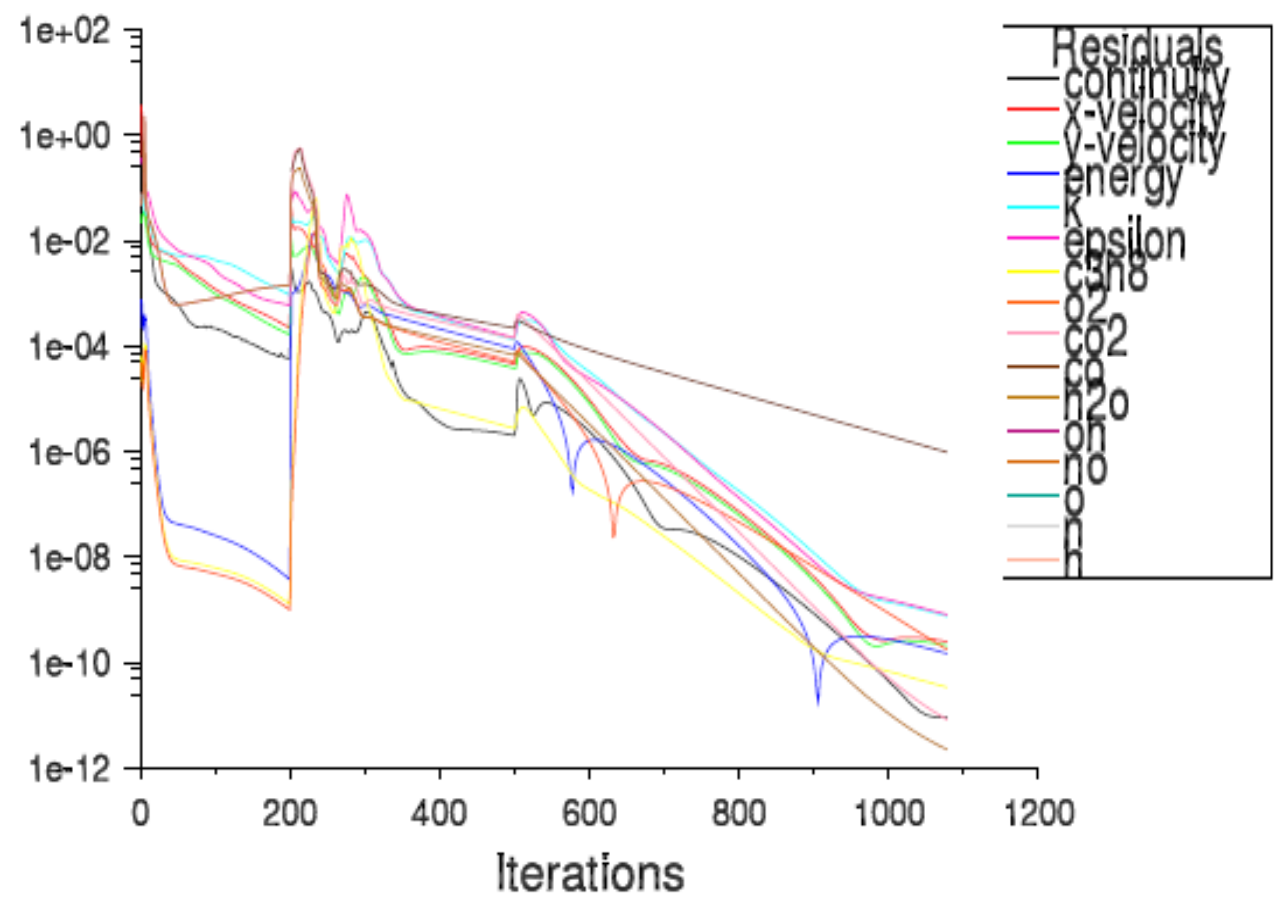

Fig. 4. Residual after 200, 500, and 1100 iterations.

At the end of the cold-flow computations, that is after the first 200 iterations, combustion was initiated as described in section 5.5c. Notice the significant jump in the value of the residuals as reminiscent of a step function when the solution adjusts to the new temperature $\left(1500^{\circ} \mathrm{C}\right.$ - ignition source) representation. The solution then converged after about 900 additional iterations. This observation is clearly depicted in Figs. 3 and 4.

Figure 5 shows the predicted velocity vector field with variable coefficient of specific heat at constant pressure. We can see from Fig. 5 that there was a high-shear flow region (jet flow) at the inlet. 
The shear flow resulted in values of velocities which range from 75 meters per second to about 142 meters per second. The strong inlet jet flow in turn created a significant level of swirl flow within the combustion chamber which is desired for obtaining excellent mixing of the propane/air mixture prior to combustion. Figure 6 shows the contours of the computed stream function. The figure shows that the eye of vortex is slightly displaced from the geometric center of the combustion chamber. This would suggest a good location of where to site our ignition source.

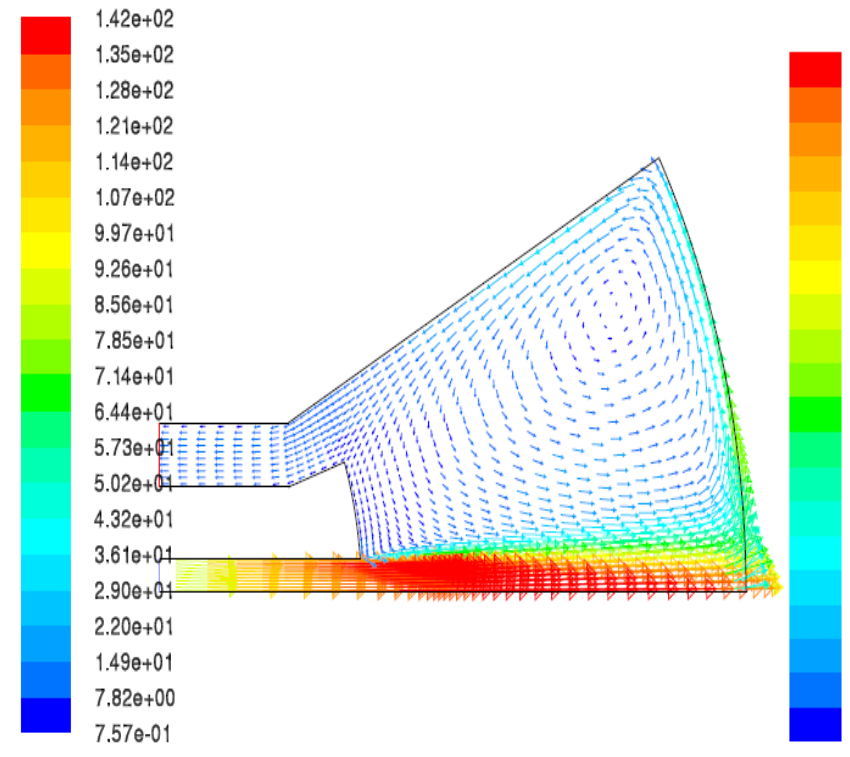

Fig. 5. Predicted flow-field velocity vector.

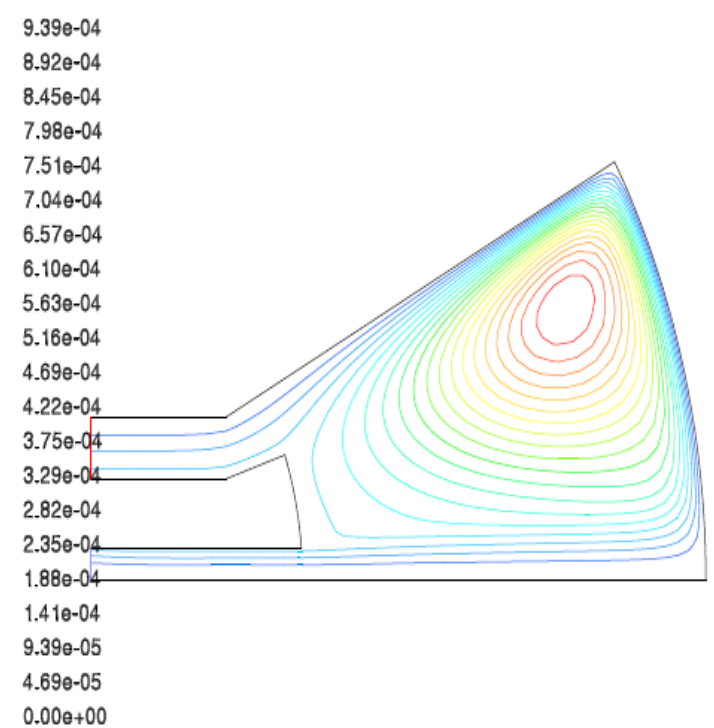

Fig. 6. Stream function contour.

Figure 7 depicts the computed contours of the static temperature field. The figure shows that the combustion efficiency of the chamber was very high as indicated by the temperature field. The predicted temperature field was found to vary from $750^{\circ} \mathrm{C}$ at the inlet to $1660^{\circ} \mathrm{C}$ during full combustion. This value compares very well with the computed flame temperature $\left(1800^{\circ} \mathrm{C}\right)$ of propane/air mixture of Ko, et al [19]. However, the computed maximum flame temperature underestimates the adiabatic flame temperature $\left(1980^{\circ} C\right)$ of premixed propane/air mixture by about $16 \%$. Also notice that the highest temperatures occur where the level of swirl was highest. 


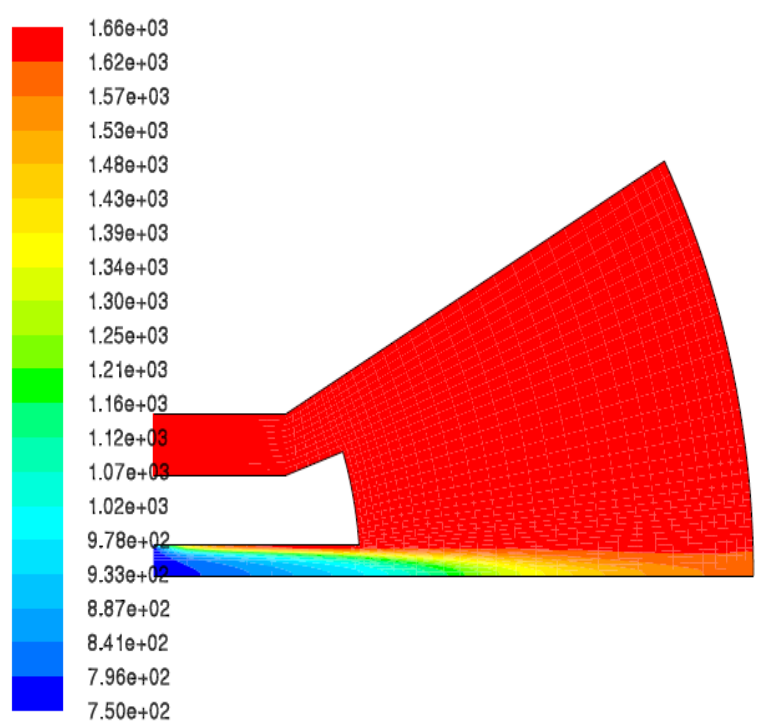

Fig. 7. Contour of static temperature.

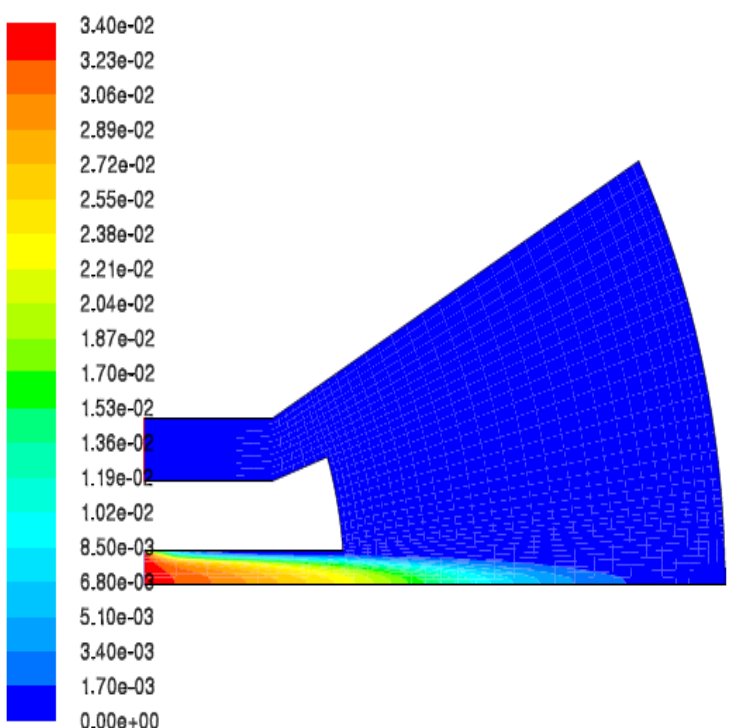

Fig. 8. Mass fraction $\mathrm{C}_{3} \mathrm{H}_{8}$.

Figure 8 above shows the calculated contours of the mass fraction of propane. We can see from Fig. 8 that the bulk of the propane was consumed within the combustion chamber and none was lost to the exhaust gases. Figure 9 shows the calculated contours of the mass fraction of carbon dioxide. We can see from Figs. 8 and 9 that there is a positive correlation, that is, the more propane that was burned the greater the amount of carbon dioxide produced. Figures 10 and 11 show the mass fractions of carbon monoxide and water produced during the combustion of premixed propane-air mixture respectively. We can again see the high correlation between Figs. 8, 9, 10 and 11. These are the natural phenomena to be expected from the combustion of a hydrocarbon with air, that is, the more the hydrocarbon that is burned the more the $\mathrm{CO}, \mathrm{CO}_{2}$ and $\mathrm{H}_{2} \mathrm{O}$ that is produced.

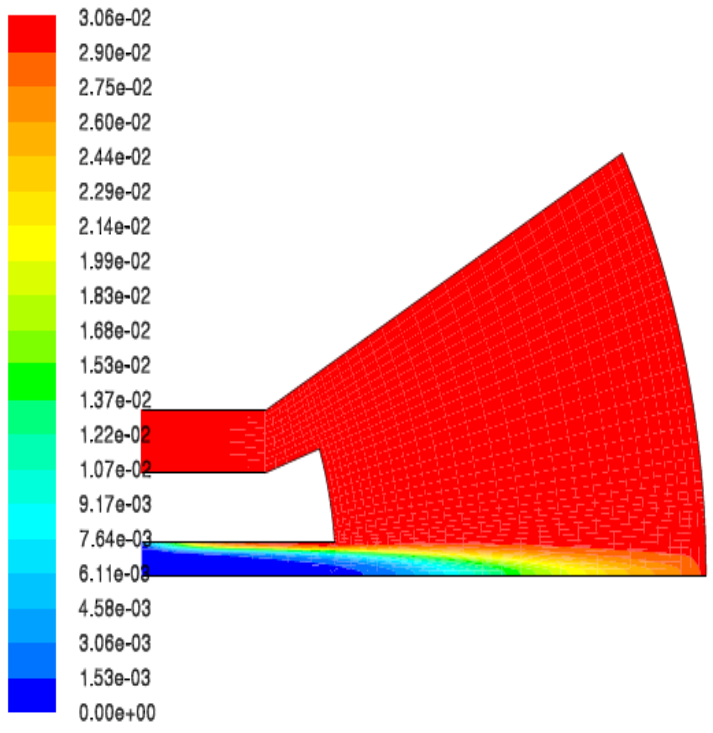

Fig. 9. Contour of mass fraction for $\mathrm{CO}_{2}$.

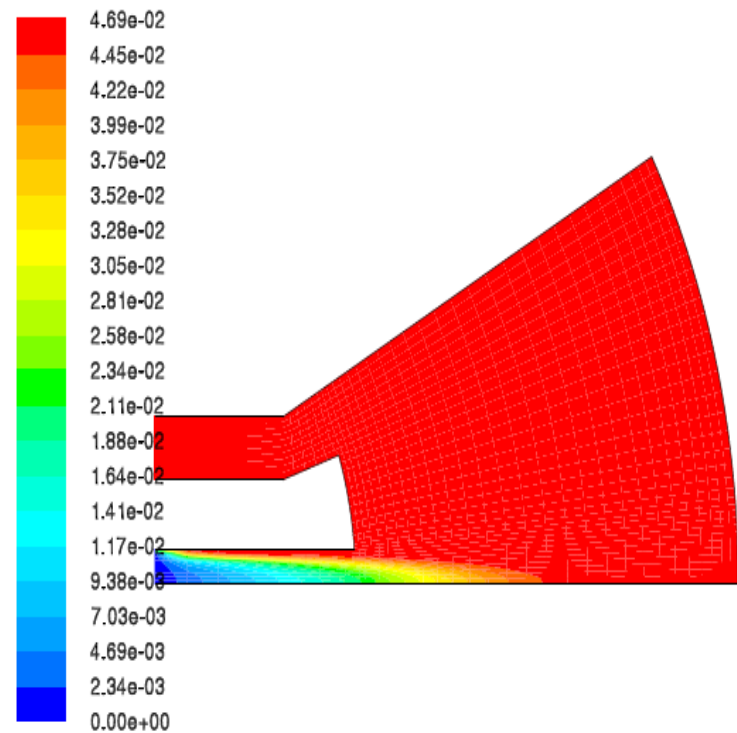

Fig. 10. Contour of mass fraction for CO.

Figure 12 shows the plot of $y^{*}$. From Fig. 12 we see that within the interior of the combustion chamber the value of $y^{*}=0$ as is to be expected. However, at regions close to the walls, we notice that the values $y^{*}$ of lies within $30<y^{*}<300$, this observation confirms that our choice of mesh density at the near wall region was adequate/accurate. 


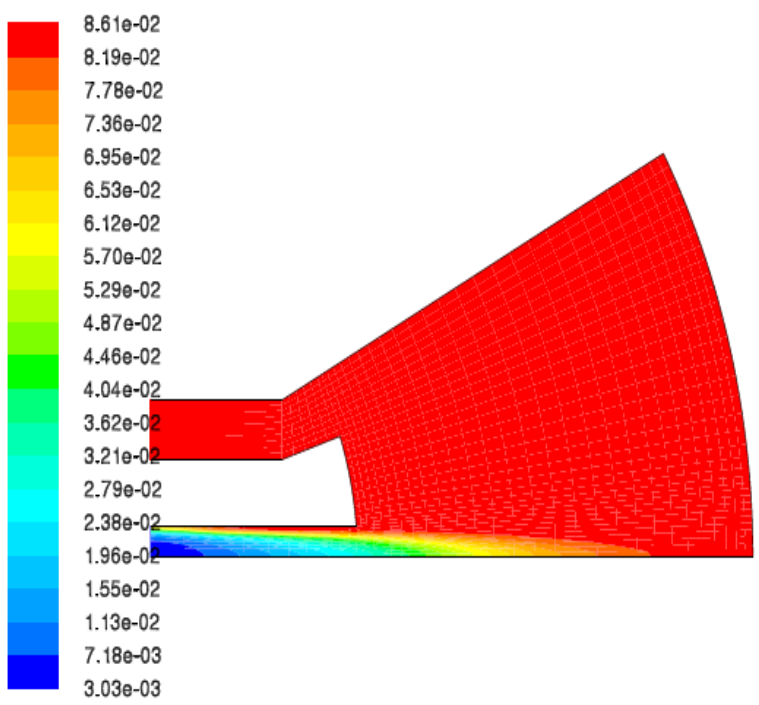

Fig. 11. Contour of molar fraction for $\mathrm{H}_{2} \mathrm{O}$.

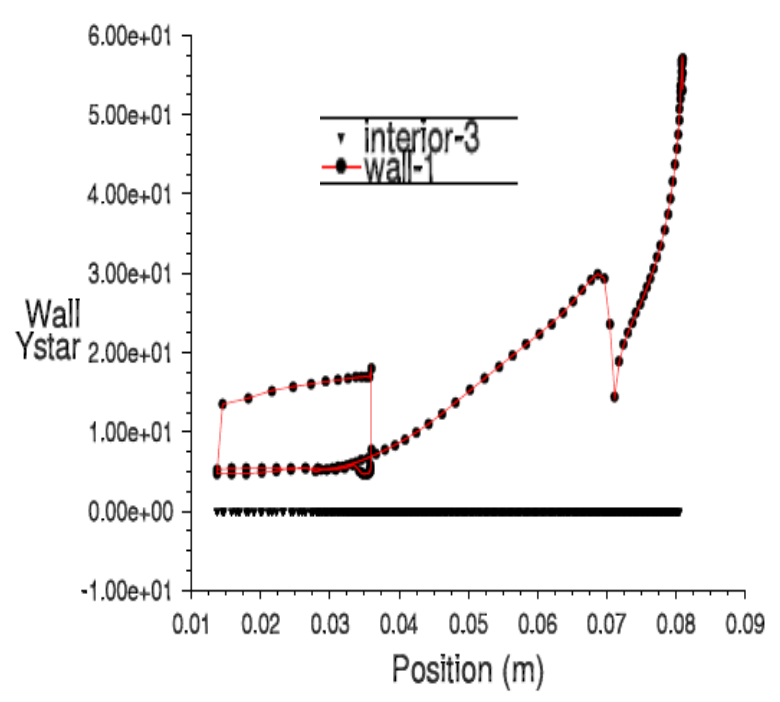

Fig. 12. $y^{*}$ distribution.

Figures 13 and 14 show the predicted contours of production and dissipation rates of turbulent kinetic energy respectively. Figure 13 shows that the bulk of turbulence was generated by two mechanisms, namely, the inlet jet (shear flow) as the premixed propane-air mixture flows into the combustion chamber and by the swirl motion that was generated within the combustion chamber. Figures 13 and 14 show that the predicted values of turbulent kinetic energy production and turbulent kinetic energy dissipation rate are of the same order of magnitude $\left(\sim 10^{5}-10^{7}\right)$, however, the dissipation rate was occurring at approximately two and a half times as fast as the production of turbulence kinetic energy. This could be attributed to the anisotropy which was prevalent in the flow.

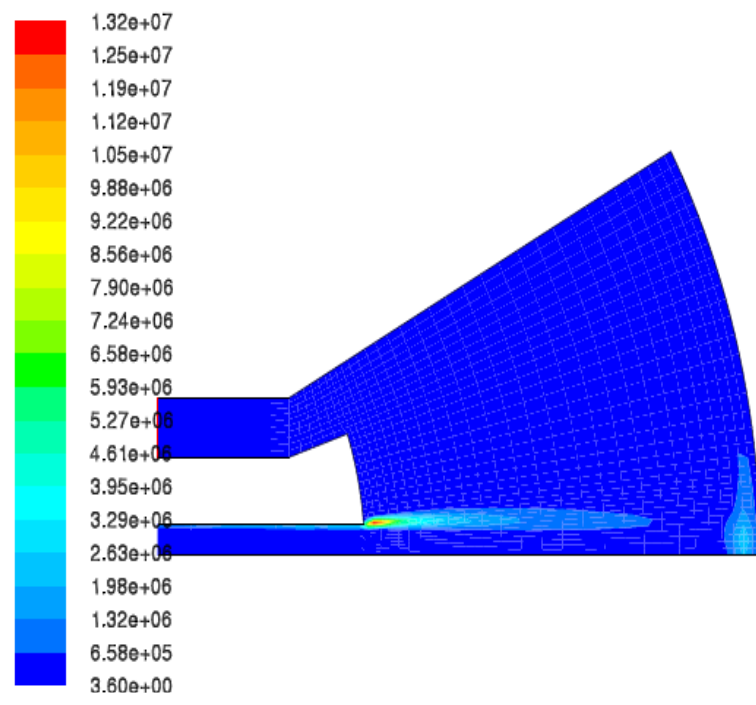

Fig. 13. Turbulence kinetic production.

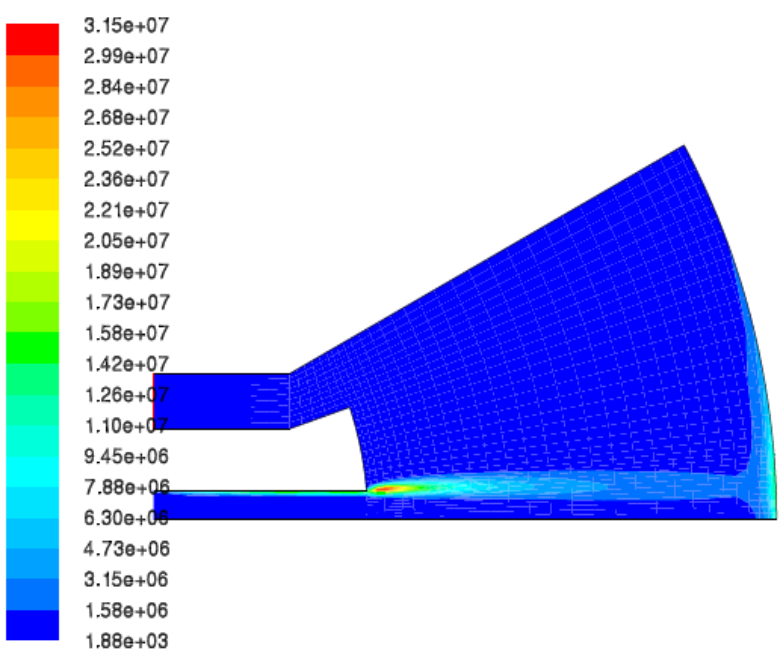

Fig. 14. Turbulent kinetic energy dissipation rate.

\section{Summary and Conclusion}

In this study we used the ANSYS Fluent CFD package to model premixed propane-air mixture oxidation in a conical reactor using the model based on the work published by Westbrook and Dryer [14]. This simplified model consists of 5 chemical (forward and backward) reactions and 12 species. The $k-\varepsilon$ model was used to model the effects of turbulence. The flow field quantities such as the velocity vector, stream function, static temperature, production and dissipation rates of turbulent kinetic 
energy that have significant influence on combustion were investigated with the present computational model. The mass fraction of propane, carbon dioxide, carbon monoxide and water vapor which give a measure of the combustion efficiency/effectiveness of the combustion chamber were studied as well. It was found that the computed peak flame temperature under estimated the adiabatic flame temperature $\left(1980^{\circ} C\right)$ of premixed propane-air mixture by about $16 \%$. The highest temperatures were found to occur where the level of swirl was highest. For this model, the computed values of the mass fractions of the following species, $\mathrm{C}_{3} \mathrm{H}_{8}, \mathrm{CO}_{2}, \mathrm{CO}$ and $\mathrm{H}_{2} \mathrm{O}$ were generally found to be in qualitative agreement with measured values presented in [20]. The computed values of $y *$ show that the computational mesh design for this study was adequate for this investigation.

Although experimental data for the combustor modeled does not exist, CFD modeling of this geometry could aid in the development of industrial burners. Furthermore, the results presented in this study appear promising, in that they could provide added impetus for the formulation of new kinetic schemes for premixed fuel-air mixtures or for the design and development of chemical reactor networks (CRNs).

\section{Acknowledgments}

We would like to acknowledge with thanks the computational resources that were generously provided by the Texas Southern University, Houston, Texas, USA.

\section{References}

[1] K. He, I. P. Androulakis, and M. G. Ierapetritou, "Multi-element flux analysis for the incorporation of detailed kinetic mechanisms in reactive simulations," Energy and Fuels, vol. 24, pp. 309-317, 2010.

[2] N. Peters and F. A. Williams, "The asymptotic structure of stoichiometric methaneair flames," Combustion and Flame, vol. 68, pp. 185-207, 1987.

[3] U. Maas and S. B. Pope, "Simplifying chemical kinetics: Intrinsic low-dimensional manifolds in composition space," Combustion and Flame, vol. 88, pp. 239-264, 1992.

[4] S. H. Lam and D. A. Goussis, "CSP method for simplifying kinetics," International Journal of Chemical Kinetics, vol. 26, pp. 461-486, 1994.

[5] L. Petzold and W. Zhu, "Model reduction for chemical kinetics: An optimization approach," AIChE Journal, vol. 45, pp. 869-886, 1999.

[6] K. Edwards and T. F. Edgar, "Reaction set simplification using variable selection techniques," Chemical Engineering Science, vol. 55, pp. 551-572, 2000.

[7] I. P. Androulakis, "Kinetic mechanism reduction based on an integer programming approach," AIChE Journal, vol. 46, pp. 361-371, 2000.

[8] O. Herbinet, W. J. Pitz, and C. K. Westbrook, "Detailed chemical kinetic mechanism for the oxidation of biodiesel fuels blend surrogate," Combustion and Flame, vol. 157, pp. 893-908, 2010.

[9] Y. Shi, H. W. Ge, J. L. Brakora, and R. D. Reitz, "Automatic chemistry mechanism reduction of hydrocarbon fuels for HCCI engines based on DRGEP and PCA methods with error control," Energy and Fuels, vol. 24, pp. 1646-1654, 2010.

[10] T. Lu and C. K. Law, "A directed relation graph method for mechanism reduction," 2005, pp. 1333-1341.

[11] D. J. Hautman, F. L. Dryer, K. P. Schug, and I. Glassman, "Multiple-step overall kinetic mechanism for the oxidation of hydrocarbons," Combustion science and technology, vol. 25, pp. 219-235, 1981.

[12] T. Kagwanpongpan and H. J. Krautz, "Numerical simulation of lignite combustion in $\mathrm{O}_{2}-\mathrm{CO}_{2}$ environment by eddy-dissipation model," in $1^{\text {st }}$ International Oxyfuel Combustion Conference 2009, Radisson SAS Hotel, Cottbus, Germany, 7-11 Sept. 2009.

[13] ANSYS Fluent, Release 12.0, ANSYS Inc., 29 Jan. 2009.

[14] C. K. Westbrook and F. L. Dryer, "Simplified reaction mechanisms for the oxidation of hydrocarbon fuels in flames," Combustion science and technology, vol. 27, pp. 31-43, 1981. 
[15] B. F. Magnussen and B. H. Hjertager, "On mathematical modeling of turbulent combustion with special emphasis on soot formation and combustion," Symposium (International) on Combustion, vol. 16, pp. 719-729, 1977.

[16] B. E. Launder and D. B. Spalding, "The numerical computation of turbulent flows," Computer Methods in Applied Mechanics and Engineering, vol. 3, pp. 269-289, 1974.

[17] D. B. Spalding, "Mixing and chemical reaction in steady confined turbulent flames," Symposium (International) on Combustion, vol. 13, pp. 649-657, 1971.

[18] J. Boussinesq, "Théorie de l'Écoulement Tourbillant," Mem. Présentés par Divers Savants Acad. Sci. Inst. Fr., vol. 23, pp. 46-50, 1877.

[19] Y. Ko, V. S. Arpaci, and R. W. Anderson, "Spark ignition of propane-air mixtures near the minimum ignition energy: Part II. A model development," Combustion and Flame, vol. 83, pp. 88-105, 1991.

[20] H. J. Curran, T. M. Jayaweera, W. J. Pitz, C. K. Westbrook, "A detailed modeling study of propane oxidation," Western States Section of the Combustion Institute 2004 Spring Meeting, Davis, CA, United States, 29-30 Mar. 2004. 